\title{
Instability of Dwell-Time Constrained Switched Nonlinear Systems
}

\author{
Matteo Della Rossa Aneel Tanwani *
}

January 7,2022

\begin{abstract}
Analyzing the stability of switched nonlinear systems under dwell-time constraints, this article investigates different scenarios where all the subsystems have a common globally asymptotically stable (GAS) equilibrium, but for the switched system, the equilibrium is not uniformly GAS for arbitrarily values of dwell-time. We motivate our study with the help of examples showing that, if near the origin all the vector fields decay at a rate slower than the linear vector fields, then the trajectories are ultimately bounded for large enough dwell-time. On the other hand, if away from the origin, the vector fields do not grow as fast as the linear vector fields, then we can only guarantee local asymptotic stability for large enough dwell-times, with region of attraction depending on the dwell-time itself. We formalize our observations for homogeneous systems, and show that, even if origin is not uniformly GAS with dwell-time switching for nonlinear systems, it still holds that the trajectories starting from a bounded set converge to a neighborhood of the origin if the dwell-time is large enough.
\end{abstract}

\section{Introduction}

Stability analysis of switched systems has been a widely studied topic in the literature over the past two decades. Depending upon the assumptions on the vector fields of individual subsystems, and the class of switching signals under consideration, several techniques have been developed, as reviewed in classical references as $[15,16,22]$. One of the basic settings in these works is to consider a switched systems with asymptotically stable subsystems (sharing a common equilibrium). Since the switching from one subsystem to another may introduce some transients which move the state trajectory away from the equilibrium for a short time period, the trajectories of the switched system eventually converge to the origin if we restrict the switching frequency. The dwell-time based stability conditions, introduced firstly in [19], formalize this idea by providing lower bound on the duration between two consecutive switches. For possible extensions of this idea for some related classes of switching signals, see [12]. From a computational viewpoint, the references $[4,6,10]$ provide some algorithms for calculating lower bounds on the dwell-time in the linear case; for the non-linear setting see the overview given in [15, Chapter 3.2] or [14, 18, 24] and references therein.

In the classical setup on dwell-time based stability conditions, it is assumed that the individual subsystems have exponentially stable equilibrium, and thus admit an exponentially decreasing Lyapunov function. It is further assumed that these Lyapunov functions are compatible with each other, in the sense that the maximum growth in the value of Lyapunov functions at switching times is bounded by a linear function. The lower bound on the dwell-time is then derived in terms of the smallest exponential decay rate among the individual Lyapunov functions, and the maximum growth rate in the value of Lyapunov functions at switching times. In particular, the existence of mutually compatible exponentially decaying Lyapunov functions implies that the switched system is uniformly exponentially stable with respect to all the switching signals satisfying a finite lower bound [23]. Some extensions of this multiple Lyapunov functions approach to provide lower

${ }^{*}$ M. Della Rossa is affiliated with UCLouvain, (Louvain-La-Neuve, Belgium). E-mail: matteo.dellarossa@uclouvain.be. A. Tanwani is affiliated with LAAS - CNRS, University of Toulouse, France.

This work was partially supported by the ANR project CONVAN with grant number ANR-17-CE40-0019-01. 
bounds on stabilizing dwell-time constraints are provided in $[12,24,25]$ and references therein. It is natural to ask the question whether such a claim holds if the individual subsystems only admit an asymptotically stable equilibrium instead of an exponentially stable one, where the growth in the value of Lyapunov function does not necessarily respect a linear bound. Such questions have been investigated in some recent references $[17,18,24]$ in the context of input-to-state stability analysis. On one hand, the papers $[18,24]$ provide sufficient conditions and a formula for calculating the lower bound on dwell-time for uniform asymptotic stability, and on the other hand, [17] provides an example of a switched system with asymptotically stable subsystems, where for each finite value of dwell-time, there exists an initial condition such that the resulting trajectory grows unbounded. Thus, one can not expect asymptotic stability for dwell-time switched systems when the individual subsystems are not exponentially stable.

In this article, we investigate stability related questions for switched systems where the subsystems only admit an asymptotically stable equilibrium, where the corresponding Lyapunov functions do not necessarily satisfy a linear growth condition at switching times. We analyze stability for dwell-time constrained switching signals (which can be arbitrarily large) and show that uniform asymptotic stability of the origin is not possible in such cases. While we lose global asymptotic stability in general, we study different stability notions, and we present corresponding stability results that arise depending on the growth rate of the vector field. With respect to the cited existing literature, the novelty our our work lies in the fact that the (in)stability results are proven without resorting any (multiple) Lyapunov functions construction, and moreover they hold for a large class of nonlinear systems and for any dwell-time threshold. We use the framework of homogeneous systems to make formal statements, which allows us to use time-scaling arguments in our analysis. The possible applications of this framework are multiple: from one side it has been shown that (non-linear) switched systems provide a modeling tool for several physical/engineering phenomena (see [15], [22] and references therein), while it is known that homogeneous systems provide a natural framework for the problem of finite-time (or practical) stabilization, see [21, 3, 20, 2].

The rest of the paper is organized as follows: In Section 2 we provide a motivation examples together with some preliminaries of switched systems, while in Section 3 recall some results from homogeneous systems literature. In Section 4 we provide our main technical results, and in Section 5 we illustrate some possible extensions in broader settings. In Section 6 we propose some concluding remarks.

\section{Preliminaries and Motivation}

Given an index set $\mathcal{I}:=\{1, \cdots, N\}$ and a finite family of vector fields $\mathcal{F}:=\left\{f_{i}\right\}_{i \in \mathcal{I}} \subset \mathcal{C}^{0}\left(\mathbb{R}^{n} \backslash\{0\}, \mathbb{R}^{n}\right)$, we study the switched system

$$
\dot{x}(t)=f_{\sigma(t)}(x(t)),
$$

where $\sigma \in \mathcal{S}:=\left\{\sigma: \mathbb{R}_{\geq 0} \rightarrow \mathcal{I} \mid \sigma\right.$ piecewise constant $\}$. Without loss of generality we suppose that signals $\sigma \in \mathcal{S}$ are right-continuous. We recall that piecewise constancy implies that $\sigma \in \mathcal{S}$ has a finite number of discontinuities in any bounded subinterval of $\mathbb{R}_{\geq 0}$. For $\sigma \in \mathcal{S}$, we denote by $\left\{t_{j}^{\sigma}\right\}$ the points at which $\sigma$ is discontinuous, also called switching instants. The set $\left\{t_{j}^{\sigma}\right\}$ may be infinite or finite, possibly reduced to the initial instant $t_{0}^{\sigma}:=0$. For a $x_{0} \in \mathbb{R}^{n}$, and a signal $\sigma \in \mathcal{S}$, we denote with $\phi_{\mathcal{F}}\left(t, x_{0}, \sigma\right)$ the solution of $(1)$ starting at $x_{0}$, evaluated at $t \geq 0$.

Definition 1. System (1) is said to be uniformly globally asymptotically stable (UGAS) on $\widehat{\mathcal{S}} \subset \mathcal{S}$, if there exists $\beta \in \mathcal{K} \mathcal{L}$ such that ${ }^{1}$

$$
\left|\phi_{\mathcal{F}}\left(t, x_{0}, \sigma\right)\right| \leq \beta\left(\left|x_{0}\right|, t\right),
$$

for all $\sigma \in \widehat{\mathcal{S}}$, for all $x_{0} \in \mathbb{R}^{n}$ and for all $t \geq 0$.

\footnotetext{
${ }^{1} \mathrm{~A}$ function $\alpha: \mathbb{R}_{>0} \rightarrow \mathbb{R}_{>0}$ is of class $\mathcal{K}(\alpha \in \mathcal{K})$ if it is continuous, $\alpha(0)=0$, and strictly increasing; it is of class $\mathcal{K}_{\infty}$ if, in addition, it is unbounded. A function $\beta: \mathbb{R}_{\geq 0} \times \mathbb{R}_{\geq 0} \rightarrow \mathbb{R}_{\geq 0}$ is of class $\mathcal{K} \mathcal{L}$ if $\beta(\cdot, s)$ is of class $\mathcal{K}$ for all $s$, and $\beta(r, \cdot)$ is decreasing with $\beta(r, s) \rightarrow 0$ as $s \rightarrow \infty$, for all $r$.
} 
by

In this article, we will consider the subclass of dwell-time switching signals; for a fixed $\tau>0$, we denote

$$
\mathcal{S}_{\mathrm{dw}}(\tau):=\left\{\sigma \in \mathcal{S} \mid t_{j}^{\sigma}-t_{j-1}^{\sigma} \geq \tau, \forall t_{j}^{\sigma}>0\right\},
$$

the class of switching signal with dwell time $\tau$.

We formally introduce here the assumption imposed on the vector field of each subsystem, $f_{i} \in \mathcal{F}$.

Assumption 1. For each $i \in \mathcal{I}, f_{i}: \mathbb{R}^{n} \rightarrow \mathbb{R}^{n}$ satisfies:

1. $f_{i} \in \mathcal{C}^{1}\left(\mathbb{R}^{n} \backslash\{0\}, \mathbb{R}^{n}\right)$

2. $f_{i}(0)=0$ and $f_{i}(x) \neq 0$ for all $x \in \mathbb{R}^{n} \backslash\{0\}$,

3. The system $\dot{x}=f_{i}(x), x(0) \in \mathbb{R}^{n}$, exhibits existence, forward uniqueness and forward completeness of solutions from any initial condition.

Remark 1. While the notion of forward completeness is standard, with forward uniqueness we mean that, for every $x_{0} \in \mathbb{R}^{n}$, if $\phi^{\prime}: \operatorname{dom}\left(\phi^{\prime}\right) \rightarrow \mathbb{R}^{n}$ and $\phi^{\prime \prime}: \operatorname{dom}\left(\phi^{\prime \prime}\right) \rightarrow \mathbb{R}^{n}$ are solutions of the initial value problem $\dot{x}(t)=f(x(t)), x(0)=x_{0}$, then $\phi^{\prime}(t)=\phi^{\prime \prime}(t)$ for all $t \in \operatorname{dom}\left(\phi^{\prime}\right) \cap \operatorname{dom}\left(\phi^{\prime \prime}\right) \cap \mathbb{R}_{\geq 0}$.

\subsection{Motivating Example}

In what follows, we will show that in the general non-linear case, global asymptotic stability of every subsystem $\dot{x}=f_{i}(x), i \in \mathcal{I}$ does not generally imply the existence of a finite $\tau>0$ such that system (1) is UGAS on $\mathcal{S}_{\mathrm{dw}}(\tau)$. To have an intuitive idea of "what goes wrong", we present a planar system, obtained by modifying a celebrated example proposed in [15, Page 19 or Example 2.1]. While the construction proposed in our example is new, one can also look at the examples in [17, Section III] in the context of i-ISS of switched systems for similar observations concerning the asymptotic behavior of the switched system.

Example 1. Consider $\mathcal{A}=\left\{A_{1}, A_{2}\right\} \subset \mathbb{R}^{2 \times 2}$ defined by $A_{1}:=\left(\begin{array}{cc}-0.2 & -1 \\ 4 & -0.2\end{array}\right)$ and $A_{2}:=\left(\begin{array}{cc}-0.2 & -4 \\ 1 & -0.2\end{array}\right)$. Given $k \in \mathbb{R}$, we define $f_{i}^{k}: \mathbb{R}^{2} \rightarrow \mathbb{R}^{2}, i \in\{1,2\}$ by

$$
f_{i}^{k}(x):=\left|A_{i} x\right|^{k-1} A_{i} x, \text { for } x \neq \mathbb{R}^{2} \backslash\{0\}
$$

and $f_{i}^{k}(0)=0$. Since the matrices $\left\{A_{i}\right\}_{i=1,2}$ are Hurwitz, and the functions $x \mapsto f_{i}^{k}(x)$ in (3) are defined as a positive scalar multiplication of the linear maps $x \mapsto A_{i} x$, the dynamical systems defined by $\dot{x}=f_{i}^{k}(x)$ are globally asymptotically stable, for $i \in\{1,2\}$ and any $k \in \mathbb{R}$. Fixing a $k \in \mathbb{R}$, we want to study the stability properties of the switched system defined by

$$
\dot{x}(t)=f_{\sigma(t)}^{k}(x(t)),
$$

considering switching signals $\sigma \in \mathcal{S}$. Let us consider, first, the simplest case: $k=1$, i.e. the linear switched system

$$
\dot{x}(t)=A_{\sigma(t)} x(t) .
$$

As shown in [15, Page 19], system (5) is not UGAS on $\mathcal{S}$. More specifically, it can be seen that there exist unbounded trajectories of (5), i.e. there exist $x_{0} \in \mathbb{R}^{n}$ and $\sigma \in \mathcal{S} \operatorname{such}$ that $\liminf _{t \rightarrow \infty}\left|\phi\left(t, x_{0}, \sigma\right)\right|=+\infty$. On the other hand, following the idea of [15, Theorem 3.2] it is easy to show that (5) is UGAS on $\mathcal{S}_{\mathrm{dw}}(\tau)$ for $\tau$ large enough.

Considering now the general case $k \neq 1$, we note first that (4) is not UGAS on $\mathcal{S}$, since system (5) is not. To describe the asymptotic behavior of system (4) in the cases $k<1$ and $k>1$ on the class of dwell-time switching signals, we consider, without loss of generality, $k=\frac{1}{2}$ and $k=2$.

Case $k=2$ : In this case, we show that for any $\tau>0$, there exist $x_{0} \in \mathbb{R}^{n}$ and $\sigma \in \mathcal{S}_{\mathrm{dw}}(\tau)$ such that the property $\liminf \operatorname{in}_{t \rightarrow+\infty}\left|\phi\left(t, x_{0}, \sigma\right)\right|>0$ holds, that is, we can find solutions which do not converge to the origin. To this end, consider, without loss of generality, the initial point $x_{0}=\left[\begin{array}{ll}1 & 0\end{array}\right]^{\top}$. Now, consider $T_{1}, T_{2}>0$ such that

$$
\phi_{f_{1}}\left(T_{1}, x_{0}\right)=\phi_{f_{2}}\left(-T_{2}, x_{0}\right)
$$



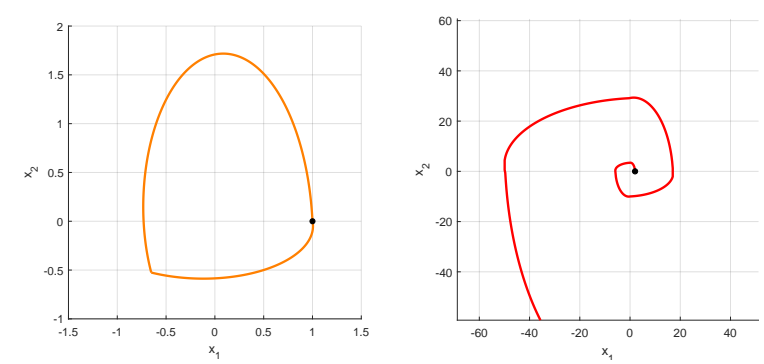

(a) Trajectory of (4) with $k=(\mathrm{b})$ Trajectory of (4) with $k=$ 2 . $1 / 2$.

Figure 1: Particular solutions of (4). In particular, for the specific switching rules defined in Example 1, note the periodic non-converging solution in the super-linear case $(k=2)$ and the diverging solution in the sub-linear case $\left(k=\frac{1}{2}\right)$.

where $\phi_{f_{i}}\left(\cdot, x_{0}\right): \mathbb{R} \rightarrow \mathbb{R}^{n}$ denotes the solution of subsystem $\dot{x}=f_{i}^{2}(x)$ starting at $x_{0}$. Intuitively, $T_{1}, T_{2}$ represent some times for which the forward solution of $\dot{x}=f_{1}^{2}(x)$ starting at $x_{0}$ "meets" the backward solution of $\dot{x}=f_{2}^{2}(x)$ starting at $x_{0}$. Let us consider the periodic switching rule (of period $\left.T_{1}+T_{2}\right)$, $\sigma: \mathbb{R}_{\geq 0} \rightarrow\{1,2\}$ defined by

$$
\sigma(t)= \begin{cases}1, & \text { if } t \in\left[0, T_{1}\right), \\ 2, & \text { if } t \in\left[T_{1}, T_{1}+T_{2}\right) .\end{cases}
$$

It holds that $\sigma \in \mathcal{S}_{\mathrm{dw}}(\widetilde{\tau})$ with $\widetilde{\tau}:=\min \left\{T_{1}, T_{2}\right\}$ and, more importantly, $\phi_{\mathcal{F}}\left(\cdot, x_{0}, \sigma\right): \mathbb{R}_{\geq 0} \rightarrow \mathbb{R}^{n}$ is periodic. From $(6), \phi_{\mathcal{F}}\left(j\left(T_{1}+T_{2}\right), x_{0}, \sigma\right)=x_{0}$, for any $j \in \mathbb{N}$, i.e. $\phi_{\mathcal{F}}\left(\cdot, x_{0}, \sigma\right): \mathbb{R}_{\geq 0} \rightarrow \mathbb{R}^{n}$ is not converging to 0 , see Figure 1a for an illustration by simulation. Considering a generic $\tau>0$, it suffices to consider a $\lambda>0$ such that $\frac{T_{i}}{\lambda^{2}}>\tau$ for any $i \in\{1,2\}, z_{0}:=\lambda x_{0}$ and the switching signal

$$
\sigma^{\prime}(t):= \begin{cases}1, & \text { if } t \in\left[0, T_{1}^{\prime}\right), \\ 2, & \text { if } t \in\left[T_{1}^{\prime}, T_{1}^{\prime}+T_{2}^{\prime}\right),\end{cases}
$$

with $T_{i}^{\prime}:=\frac{T_{i}}{\lambda^{2}}$, for any $i \in\{1,2\}$. With this definition, it is clear that $\sigma^{\prime} \in \mathcal{S}_{\mathrm{dw}}(\tau)$ and it can be seen that $\phi\left(\cdot, z_{0}, \sigma^{\prime}\right)$ is, once again, periodic and not-converging.

Case $k=1 / 2$ : In this case it can be shown that, for any $\tau>0$, there exists $x_{0} \in \mathbb{R}^{n}$ with $\left|x_{0}\right|$ large enough and $\sigma \in \mathcal{S}_{\mathrm{dw}}(\tau)$ such that $\lim _{t \rightarrow+\infty}\left|\phi_{\mathcal{F}}\left(t, x_{0}, \sigma\right)\right|=+\infty$, that is, there exist diverging solutions of (4). The formal definition of such "pathological" $x_{0}$ and $\sigma$ is avoided here, since a similar construction has been recently proposed in [17, Section 3] for a slightly different (but qualitatively equivalent) example. A graphical representation of a particular diverging solution is presented in Figure $1 \mathrm{~b}$.

\subsection{Our Methodology}

Inspired by our motivating example, our basic idea for the stability analysis of (1) is to compare its solutions with the solutions of another system obtained by applying a particular nonlinear transformation of the vector fields. By formulating simpler questions for the transformed system, one can then deduce information about the qualitative behavior of the original system. To follow this roadmap, we will work with the following assumption:

Assumption 2 (Homogeneity). For each $i \in \mathcal{I}$, the function $f_{i}: \mathbb{R}^{n} \rightarrow \mathbb{R}^{n}$, is homogeneous of degree $k \in \mathbb{R}$, that is,

$$
f_{i}(\lambda x)=\lambda^{k} f_{i}(x), \quad \forall x \in \mathbb{R}^{n}, \forall \lambda>0 .
$$

If $f_{i}: \mathbb{R}^{n} \rightarrow \mathbb{R}^{n}$ satisfies (7), we write $f_{i} \in \mathcal{H}_{n}^{k}$. 
Remark 2. In the context of dynamical systems, more general notions of homogeneity are often considered, see for example [2], [11, Definition 3.8], [21, Theorem 2]. For a thorough historical discussion on "homogeneity notions", we refer to the monograph [20]. It is worth noting that there always exists a change of coordinates which transforms any "generalized" homogeneous system (in the sense of $[2,11,21]$ ) to a system satisfying Assumption 2, see [20, Theorem 8.9], and thus the subsequent results can be generalized for these more general notions of homogeneity. $1 "$.

Given $f \in \mathcal{H}_{n}^{k}$, we define in what follows a transformation which can be seen as a "reduction of degree

Definition 2 (Reduction). Consider $f \in \mathcal{H}_{n}^{k}$, satisfying Assumption 1. We define the reduction of degree 1 of $f$ as the function $g_{f} \in \mathcal{H}_{n}^{1}$ defined by $g_{f}(0)=0$ and

$$
g_{f}(x):=\frac{|f(x)|^{\frac{1}{k}}}{|f(x)|} f(x), \quad \forall x \in \mathbb{R}^{n} \backslash\{0\},
$$

if $k \neq 0$, and by $g_{f}(x):=|x| \frac{f(x)}{|f(x)|}$ if $k=0$.

When clear from the context, we write $g$ instead of $g_{f}$. In the case $k \neq 0$, we have the property that $g_{f}(x)=f(x)$, on the set $\mathcal{C}_{f}:=\left\{x \in \mathbb{R}^{n}|| f(x) \mid=1\right\}$, i.e. we are not modifying $f$ on a closed hypersurface surrounding the origin. Then, $g_{f}$ is defined simply as a "dilation" of degree 1 of $f$. In particular, if $f$ is homogeneous of degree 1 then $f \equiv g_{f}$. The case $k=0$ (i.e. vector fields which are constant along lines) will not be treated explicitly, since it needs to be studied differently, due to the different expression of $g_{f}$ in Definition 2. The qualitative behavior of this case is completely equivalent to the ones obtained in the case $k<1, k \neq 0$.

In the context of switched systems, the usefulness of introducing the reduction of degree 1 of subsystems $\dot{x}=f_{i}(x)$, as in Definition 2, is mainly given by the following statement; which is a well-known result in the case of linear subsystems (see [19, Lemma 2]); the proof in the homogeneous of degree 1 case essentially follows the construction presented in [15, Section 3.2.1], and it is thus omitted.

Lemma 1. Consider the family of functions $\mathcal{G}=\left\{g_{i}\right\}_{i \in \mathcal{I}} \subset \mathcal{H}_{n}^{1}$ satisfying Assumption 1 and suppose that subsystem $\dot{x}=g_{i}(x)$ is GAS (and thus GES), for each $i \in \mathcal{I}$. Then there exists a $\tau_{\mathcal{G}}>0$ such that the system

$$
\dot{x}(t)=g_{\sigma(t)}(x(t))
$$

is $U G A S$ on $\mathcal{S}_{\mathrm{dw}}\left(\tau_{\mathcal{G}}\right)$.

\section{Analytical Tools for Homogeneous Systems}

Given a $k \in \mathbb{R} \backslash\{0\}$, consider a vector field $f \in \mathcal{H}_{n}^{k}$ satisfying Assumption 1; we note that the homogeneity property is inherited by every solution of the system

$$
\dot{x}=f(x) .
$$

More precisely, we have, that $\forall t \in \mathbb{R}_{\geq 0}, \forall x \in \mathbb{R}^{n}, \forall \lambda>0$,

$$
\phi_{f}(t, \lambda x)=\lambda \phi_{f}\left(\lambda^{k-1} t, x\right),
$$

where $\phi_{f}(t, x)$ denotes the solution of (9) starting at $x$ evaluated at $t \geq 0$. Moreover, we have the following characterization of stability, for the proof see [3, Corollary 5.4].

Lemma 2. Consider $f \in \mathcal{H}_{n}^{k}$ satisfying Assumption 1 and suppose that the corresponding system (9) is GAS. Then: 
- If $k=1$, the system is globally exponentially stable (GES), more precisely, there exist $M_{1}, M_{2}, \mu>0$ such that

$$
M_{1}|x| e^{-\mu t} \leq\left|\phi_{f}(t, x)\right| \leq M_{2}|x| e^{-\mu t}
$$

for any $x \in \mathbb{R}^{n}$, and any $t \geq 0$.

- If $k>1$, there exist $M_{1}, M_{2}>0$ such that

$$
M_{1}\left(1+|x|^{k-1} t\right)^{\frac{1}{1-k}}|x| \leq\left|\phi_{f}(t, x)\right| \leq M_{2}\left(1+|x|^{k-1} t\right)^{\frac{1}{1-k}}|x|,
$$

for any $x \in \mathbb{R}^{n}$, and any $t \geq 0$.

- If $k<1$, the system (9) is finite-time stable, i.e., for every $x \in \mathbb{R}^{n}$ there exists a $T(x)>0$ such that $\phi_{f}(t, x)=0$, for all $t \geq T(x)$.

Note that if $f$ satisfies Assumption 1, then $g_{f} \in \mathcal{C}^{1}\left(\mathbb{R}^{n} \backslash\{0\}, \mathbb{R}^{n}\right)$ and, defining $M:=\max _{|y|=1}|f(y)|$, it satisfies the linear growth condition $\left|g_{f}(x)\right| \leq M|x|$. Then $g_{f}$ is locally Lipschitz on $\mathbb{R}^{n}$ and in particular the system $\dot{z}=g_{f}(z)$ exhibits existence, uniqueness and completeness (backward and forward) of solutions, for any initial condition.

Lemma 3. Consider $k \neq 0$ and $f \in \mathcal{H}_{n}^{k}$ satisfying Assumption 1 and its reduction of degree $1, g_{f} \in \mathcal{H}_{n}^{1}$ as in Definition 2. Consider the dynamical systems

$$
\begin{aligned}
& \dot{x}=f(x), \\
& \dot{z}=g_{f}(z) .
\end{aligned}
$$

Denoting with $\phi_{f}$ and $\phi_{g}$ the flow maps of (11a) and (11b) respectively, define the map $\theta: \mathbb{R}_{\geq 0} \times\left(\mathbb{R}^{n} \backslash\{0\}\right) \rightarrow$ $\mathbb{R}$ by

$$
\theta(t, x)=\int_{0}^{t} \frac{\left|f\left(\phi_{g}(s, x)\right)\right|^{\frac{1}{k}}}{\left|f\left(\phi_{g}(s, x)\right)\right|} d s .
$$

Then

$$
\phi_{g}(t, x)=\phi_{f}(\theta(t, x), x), \quad \forall x \neq 0, \forall t \in \mathbb{R}_{\geq 0} .
$$

The proof essentially follows the construction proposed in [9, Lemma 2.4]. Lemma 2 and Lemma 3 imply that

$$
\text { (11a) is GAS } \Leftrightarrow(11 b) \text { is GES. }
$$

In the following we explicitly collect some remarkable properties of the time-scaling function $\theta$, defined in (12).

Proposition 1 (Properties of $\theta$ ). Consider $f \in \mathcal{H}_{n}^{k}$ with $k \neq 0$ satisfying Assumption 1. Then, the function $\theta: \mathbb{R}_{\geq 0} \times\left(\mathbb{R}^{n} \backslash\{0\}\right) \rightarrow \mathbb{R}$ in (12) satisfies

1. For each $x \in \mathbb{R}^{n} \backslash\{0\}$, and each $t \in \mathbb{R}_{\geq 0}$, the functions $\theta(\cdot, x): \mathbb{R}_{\geq 0} \rightarrow \mathbb{R}$, and $\theta(t, \cdot): \mathbb{R}^{n} \backslash\{0\} \rightarrow \mathbb{R}$ are continuous, respectively.

2. For each $x \in \mathbb{R}^{n} \backslash\{0\}$, each $t \in \mathbb{R}_{\geq 0}$, and each $\lambda>0$,

$$
\theta(t, \lambda x)=\frac{\theta(t, x)}{\lambda^{k-1}} .
$$

3. For each $x \in \mathbb{R}^{n} \backslash\{0\}, \theta(0, x)=0$ and $\theta(\cdot, x): \mathbb{R}_{\geq 0} \rightarrow \mathbb{R}$ is strictly increasing.

4. If $k=1$, then $\theta(t, x)=t, \forall t \in \mathbb{R}_{\geq 0}, \forall x \neq 0$.

4'. If $k<1$ and system (9) is GAS, then, for all $x \neq 0$, there exists $T(x)>0$ such that $\lim _{s \rightarrow+\infty} \theta(s, x)=$ $T(x)$ and $\theta(\cdot, x)$ is invertible, with inverse defined on $[0, T(x))$. 
4". If $k>1$ and system (9) is GAS, then, for all $x \in \mathbb{R}^{n} \backslash\{0\}, \lim _{s \rightarrow+\infty} \theta(s, x)=+\infty$ and $\theta(\cdot, x)$ is invertible, with inverse defined on $[0,+\infty)$.

Given any $x \in \mathbb{R}^{n} \backslash\{0\}$, we denote by $\theta^{-1}(\cdot, x)$ the inverse of $\theta$ (with respect to the first argument); note that the domain of definition depends on the homogeneity degree of $f$. Since reductions of degree 1 (Definition 2) of the subsystems $\dot{x}=f_{i}(x)$ will be used to study the behavior of switched systems as in (1), we need the following result.

Lemma 4 ("Translation" of solutions). Consider $k \neq 0$ and a finite family $\mathcal{F}=\left\{f_{i}\right\}_{i \in \mathcal{I}} \subset \mathcal{H}_{n}^{k}$ satisfying Assumption 1. Suppose that $\dot{x}=f_{i}(x)$ is GAS, for each $i \in \mathcal{I}$. Moreover, consider the set $\mathcal{G}=\left\{g_{i}\right\}_{i \in \mathcal{I}} \subset \mathcal{H}_{n}^{1}$ given by the reductions of degree 1 of the $f_{i}$, as defined in Definition 2. Given any $x_{0} \in \mathbb{R}^{n} \backslash\{0\}$ and $\sigma \in \mathcal{S}$, define

$$
T_{\sigma}\left(x_{0}\right):=\inf \left\{t>0 \mid \phi_{\mathcal{F}}\left(t, x_{0}, \sigma\right)=0\right\}
$$

with the possibility that $T_{\sigma}\left(x_{0}\right)=+\infty$. Then, there exists a $\sigma_{\mathcal{G}} \in \mathcal{S}$ and a strictly increasing $\gamma:\left[0, T_{\sigma}\left(x_{0}\right)\right) \rightarrow$ $\mathbb{R}_{\geq 0}$ with $\gamma(0)=0$, and $\lim _{t \rightarrow T\left(x_{0}\right)^{-}} \gamma(t)=+\infty$, such that

$$
\phi_{\mathcal{F}}\left(t, x_{0}, \sigma\right)=\phi_{\mathcal{G}}\left(\gamma(t), x_{0}, \sigma_{\mathcal{G}}\right), \quad \forall t \in\left[0, T_{\sigma}\left(x_{0}\right)\right) .
$$

Similarly, for any $x_{0} \in \mathbb{R}^{n} \backslash\{0\}$ and any $\sigma_{\mathcal{G}} \in \mathcal{S}$, there exists $a \in \mathcal{S}$ and a strictly increasing $\chi: \mathbb{R}_{\geq 0} \rightarrow \mathbb{R}_{\geq 0}$ with $\chi(0)=0$ and $\lim _{s \rightarrow+\infty} \chi(s)=T_{\sigma}\left(x_{0}\right)$, such that

$$
\phi_{\mathcal{G}}\left(s, x_{0}, \sigma_{\mathcal{G}}\right)=\phi_{\mathcal{F}}\left(\chi(s), x_{0}, \sigma\right), \quad \forall s \in \mathbb{R}_{\geq 0} .
$$

Let us note that, by Lemma 2 , the case $T_{\sigma}\left(x_{0}\right)<+\infty$ is possible only if $k<1$; in all the other cases the function $\gamma$ is globally defined (and, similarly, the function $\chi$ is unbounded). The proof of Proposition 1 is postponed to Appendix A.1 while the proof of Lemma 4 is postponed to Appendix A.2, to avoid breaking the flow of the presentation.

\section{Main Results}

In this section we will consider a finite family of subsystems $\mathcal{F}=\left\{f_{i}\right\}_{i \in \mathcal{I}} \subset \mathcal{H}_{n}^{k}$ satisfying Assumption 1 , and we suppose that each subsystem $\dot{x}=f_{i}(x)$ is GAS, for each $i \in \mathcal{I}$. Example 1 showed that this does not necessarily imply the uniform global asymptotic stability of the switched system (1) under (any class of) dwell-time switching signals. On the other hand, weaker results can be obtained; in particular we analyze the cases $k>1$ and $k<1$ (the case $k=1$ is already characterized by Lemma 1 ).

Theorem 1 (Case $k>1$ ). Consider a finite family of vector fields satisfying Assumption $1, \mathcal{F}=\left\{f_{i}\right\}_{i \in \mathcal{I}} \subset$ $\mathcal{H}_{n}^{k}$, with $k>1$, and suppose that $\dot{x}=f_{i}(x)$ is $G A S$, for each $i \in \mathcal{I}$. Consider the set $\mathcal{G}=\left\{g_{i}\right\}_{i \in \mathcal{I}} \subset \mathcal{H}_{n}^{1}$, where $g_{i} \equiv g_{f_{i}}$ is defined as in Definition 2. Then, the following hold:

1. (Ultimate boundedness) For every $\tau>0$ there exists an $R(\tau)>0$ such that, for each $x_{0} \in \mathbb{R}^{n}$ and $\sigma \in \mathcal{S}_{\mathrm{dw}}(\tau)$

$$
\limsup _{t \rightarrow+\infty}\left|\phi_{\mathcal{F}}\left(t, x_{0}, \sigma\right)\right| \leq R(\tau) .
$$

2. (Non-Stability) Suppose that there exist $\tau_{\mathcal{G}}^{\star}>0, \sigma_{\mathcal{G}} \in \mathcal{S}\left(\tau_{\mathcal{G}}^{\star}\right)$ and $x_{0} \in \mathbb{R}^{n}$ such that the solution of the switched system $\dot{x}=g_{\sigma_{\mathcal{G}}(t)}(x)$, denoted by $\phi_{\mathcal{G}}\left(\cdot, x_{0}, \sigma_{\mathcal{G}}\right): \mathbb{R}_{+} \rightarrow \mathbb{R}^{n}$, is diverging, i.e.

$$
\limsup _{s \rightarrow+\infty}\left|\phi_{\mathcal{G}}\left(s, x_{0}, \sigma_{\mathcal{G}}\right)\right|=+\infty \text {. }
$$

Then, for every $\tau>0$, there exists a ball $\mathbb{B}(0, \bar{R}(\tau))$, a sequence $\left(x_{0 \ell}\right)_{\ell \in \mathbb{N}}, x_{0 \ell} \rightarrow 0, \sigma_{\ell} \in \mathcal{S}_{\mathrm{dw}}(\tau)$ and $t_{\ell}>0$ such that $\phi_{\mathcal{F}}\left(t_{\ell}, x_{0 \ell}, \sigma_{\ell}\right) \notin \mathbb{B}(0, \bar{R}(\tau))$ (i.e. 0 is not Lyapunov stable). ${ }^{2}$

\footnotetext{
${ }^{2}$ We denote by $\mathbb{B}(0, r)$ the open ball of radius $r$ centered at 0 , by $\bar{B}(0, r)$ the closure of $\mathbb{B}(0, r)$ (i.e. the corresponding closed ball).
} 
Proof. We denote by $\theta_{i}: \mathbb{R} \times\left(\mathbb{R}^{n} \backslash\{0\}\right) \rightarrow \mathbb{R}$ the map defined by (12), when considering the sub-vector field $f_{i}: \mathbb{R}^{n} \rightarrow \mathbb{R}^{n}$. With $\beta_{i} \in \mathcal{K} \mathcal{L}$, we denote the function characterizing the GAS property of the subsystem $\dot{x}=f_{i}(x)$, for each $i \in \mathcal{I}$. Since, by (14), subsystem $\dot{x}=g_{i}(x)$ is GES for each $i \in \mathcal{I}$, considering the switched system

$$
\dot{x}(t)=g_{\sigma_{\mathcal{G}}(t)}(x(t)),
$$

there exists a $\tau_{\mathcal{G}}>0$, such that $(18)$ is UGAS on $\mathcal{S}_{\mathrm{dw}}\left(\tau_{\mathcal{G}}\right)$, as proved in Lemma 1 . Let us call $\beta_{\mathcal{G}} \in \mathcal{K} \mathcal{L}$ the function characterizing the UGAS property of $(18)$ on $\mathcal{S}_{\mathrm{dw}}\left(\tau_{\mathcal{G}}\right)$, as in Definition 1.

- Let us prove item 1. Fix $\tau>0$, we suppose from now on that the considered $\sigma \in \mathcal{S}_{\mathrm{dw}}(\tau)$ have an infinite number of discontinuities (i.e. switching instants), indeed if $\sigma$ has a finite number of discontinuities, we necessarily have $\lim _{t \rightarrow+\infty}\left|\phi_{\mathcal{F}}\left(t, x_{0}, \sigma\right)\right|=0$, since all subsystems are GAS.

Let us consider $R_{1}(\tau)>0$ big enough such that

$$
\theta_{i}\left(\tau_{\mathcal{G}}, x\right) \leq \tau, \quad \forall|x| \geq R_{1}(\tau), \forall i \in \mathcal{I} .
$$

Such a choice is possible recalling Proposition 1 , since, $\forall i \in \mathcal{I}, \theta_{i}\left(\tau_{\mathcal{G}}, \cdot\right): \mathbb{R}^{n} \backslash\{0\} \rightarrow \mathbb{R}$ is continuous and, as shown in $(15), \theta_{i}\left(\tau_{\mathcal{G}}, \cdot\right)$ is strictly decreasing along lines, i.e.

$$
\theta_{i}\left(\tau_{\mathcal{G}}, \lambda_{1} x\right)>\theta_{i}\left(\tau_{\mathcal{G}}, \lambda_{2} x\right), \quad \forall 0<\lambda_{1}<\lambda_{2}, \forall x \neq 0,
$$

and moreover $\lim _{\lambda \rightarrow+\infty} \theta_{i}\left(\tau_{\mathcal{G}}, \lambda x\right)=0$, for all $x \in \mathbb{R}^{n}$, see also Figure 2a. Using (19), the proof of item 1 follows from the next lemma, which will be also used in later sections.

Lemma 5. For a given $\tau>0$, consider $R_{1}(\tau)$ such that (19) holds. Consider $R_{2}(\tau)>0$ such that $R_{2}(\tau) \geq$ $\beta_{i}\left(0, R_{1}(\tau)\right), \forall i \in \mathcal{I}$ and define

$$
R(\tau):=\beta_{\mathcal{G}}\left(0, R_{2}(\tau)\right)
$$

Then item 1 holds.

Proof of Lemma 5. We proceed in two steps.

Step A: Consider $x_{0} \in \mathbb{R}^{n}$ such that $\left|x_{0}\right|>R_{1}(\tau), \sigma \in \mathcal{S}_{\mathrm{dw}}(\tau)$ and define $t_{\underline{j}}^{\sigma}:=\inf \left\{t_{j}^{\sigma}>0|| \phi_{\mathcal{F}}\left(t_{j}^{\sigma}, x_{0}, \sigma\right) \mid \leq\right.$ $\left.R_{1}(\tau)\right\}$, that is, the first switching instant for which the solution starting at $x_{0}$ is inside the ball $\overline{\mathbb{B}}\left(0, R_{1}(\tau)\right)$. We want to prove that $t_{\underline{j}}^{\sigma}$ is finite. Consider the switching signal $\sigma_{\mathcal{G}} \in \mathcal{S}$ and $\gamma: \mathbb{R}_{+} \rightarrow \mathbb{R}_{+}$, given by Lemma 4, such that

$$
\phi_{\mathcal{F}}\left(t, x_{0}, \sigma\right)=\phi_{\mathcal{G}}\left(\gamma(t), x_{0}, \sigma_{\mathcal{G}}\right), \forall t \in \mathbb{R}_{+} .
$$

Defining $x_{j}:=\phi_{\mathcal{F}}\left(t_{j}^{\sigma}, x_{0}, \sigma\right)$ for all $j \in \mathbb{N}$, by definition of $t_{\underline{j}}^{\sigma}$, we have $\left|x_{j}\right|>R_{1}(\tau)$ for all $j<\underline{j}$. This, since $\sigma \in \mathcal{S}_{\mathrm{dw}}(\tau)$ and recalling (19), implies that

$$
\theta_{\sigma\left(t_{j}^{\sigma}\right)}^{-1}\left(t_{j+1}^{\sigma}-t_{j}^{\sigma}, x_{j}\right) \geq \theta_{\sigma\left(t_{j}^{\sigma}\right)}^{-1}\left(\tau, x_{j}\right) \geq \tau_{\mathcal{G}}, \quad \forall j<\underline{j} .
$$

By the definition of $\gamma: \mathbb{R}_{+} \rightarrow \mathbb{R}_{+}$in (35) in Appendix A.2, we finally have that $t_{j+1}^{\sigma_{\mathcal{G}}}-t_{j}^{\sigma_{\mathcal{G}}}=\theta_{\sigma\left(t_{j}^{\sigma}\right)}^{-1}\left(t_{j+1}^{\sigma}-\right.$ $\left.t_{j}^{\sigma}, x_{j}\right) \geq \tau_{\mathcal{G}}$, for all $j<j$. Rephrasing, we have proved that the signal $\sigma_{\mathcal{G}}$ has a dwell time $\tau_{\mathcal{G}}$, at least until the switching instant $t_{\underline{j}}^{\sigma \overline{\mathcal{G}}}$,implying that

$$
\left|\phi_{\mathcal{F}}\left(t, x_{0}, \sigma\right)\right|=\left|\phi_{\mathcal{G}}\left(\gamma(t), x_{0}, \sigma_{\mathcal{G}}\right)\right| \leq \beta_{\mathcal{G}}\left(\gamma(t),\left|x_{0}\right|\right),
$$

for every $t \in\left[0, t_{\underline{j}}^{\sigma}\right]$. Since by definition $\lim _{t \rightarrow+\infty} \gamma(t)=+\infty$, and $\lim _{s \rightarrow+\infty} \beta_{\mathcal{G}}\left(s,\left|x_{0}\right|\right)=0$, we conclude that $t_{\underline{j}}^{\sigma}$ is finite, if not we have a contradiction. In other words, we have proved that, for any initial condition $x_{0} \notin \overline{\mathbb{B}}\left(0, R_{1}(\tau)\right)$, solutions of (1) reach $\overline{\mathbb{B}}\left(0, R_{1}(\tau)\right)$ (for a finite switching instant).

Step B: Consider any $x_{0} \in \mathbb{R}^{n}$ such that $\left|x_{0}\right| \leq R_{1}(\tau)$ and any $\sigma \in \mathcal{S}_{\mathrm{dw}}(\tau)$, consider

$$
t_{j^{\star}}^{\sigma}:=\inf \left\{t_{j}^{\sigma}>0|| \phi_{\mathcal{F}}\left(t_{j}^{\sigma}, x_{0}, \sigma\right) \mid>R_{1}(\tau)\right\} .
$$


Intuitively, $t_{j^{\star}}^{\sigma}$ is defined as the first switching instant (if any) for which the solution starting at $x_{0}$ is outside the set $\overline{\mathbb{B}}\left(0, R_{1}(\tau)\right)$, (if such switching instant does not exist, we set $t_{j^{\star}}^{\sigma}=+\infty$ ). Consider any $t_{j}^{\sigma}<t_{j^{\star}}^{\sigma}$, by definition, we have $\left|\phi_{\mathcal{F}}\left(t_{j}^{\sigma}, x_{0}, \sigma\right)\right| \leq R_{1}(\tau)$ and supposing $\sigma\left(t_{j}^{\sigma}\right)=i_{j} \in \mathcal{I}$, we have

$$
\begin{aligned}
\left|\phi_{\mathcal{F}}\left(t, x_{0}, \sigma\right)\right| & \leq \beta_{i_{j}}\left(t-t_{j}^{\sigma},\left|\phi_{\mathcal{F}}\left(t_{j}^{\sigma}, x_{0}, \sigma\right)\right|\right) \\
& \leq \beta_{i_{j}}\left(0, R_{1}(\tau)\right) \leq R_{2}(\tau),
\end{aligned}
$$

for every $t \in\left[t_{j}^{\sigma}, t_{j_{+1}}^{\sigma}\right]$. By arbitrariness of $t_{j}^{\sigma}<t_{j^{\star}}^{\sigma}$ we thus have $\left|\phi_{\mathcal{F}}\left(t, x_{0}, \sigma\right)\right| \leq R_{2}(\tau)$, for all $t \in\left[0, t_{j^{\star}}^{\sigma}\right]$. Since, by definition, $x_{j^{\star}}:=\phi_{\mathcal{F}}\left(t_{j^{\star}}^{\sigma}, x_{0}, \sigma\right)$ is such that $\left|x_{j^{\star}}\right|>R_{1}(\tau)$, reasoning as in (21) in Step A, it is easy to see that

$$
\left|\phi_{\mathcal{F}}\left(t, x_{0}, \sigma\right)\right| \leq \beta_{\mathcal{G}}\left(0,\left|x_{j^{\star}}\right|\right)<\beta_{\mathcal{G}}\left(0, R_{2}(\tau)\right)=R(\tau), \forall t \geq t_{j^{\star}}^{\sigma} .
$$

A generic solution of (1) can be reconstructed concatenating "partial trajectories" lying in the two cases $\left(\left|x_{0}\right|>R_{1}(\tau)\right.$ and $\left.\left|x_{0}\right| \leq R_{1}(\tau)\right)$ analyzed in Steps A and B, concluding the proof of Lemma 5 .

- Let us now prove item 2: consider $\tau_{\mathcal{G}}^{\star}>0, \sigma_{\mathcal{G}} \in \mathcal{S}_{\mathrm{dw}}\left(\tau_{\mathcal{G}}^{\star}\right)$ and $x_{0} \in \mathbb{R}^{n}$ such that the corresponding solution of the reduced system (18) is diverging, i.e., more explicitly, $\forall M>0$, there exists an $s_{M} \in \mathbb{R}_{+}$such that $\left|\phi_{\mathcal{G}}\left(s_{M}, x_{0}, \sigma_{\mathcal{G}}\right)\right|>M$. For any $\lambda>0$, the solution $\phi_{\mathcal{G}}\left(\cdot, \lambda x_{0}, \sigma_{\mathcal{G}}\right): \mathbb{R}_{+} \rightarrow \mathbb{R}^{n}$ is also diverging, since $\phi_{\mathcal{G}}\left(s, \lambda x_{0}, \sigma_{\mathcal{G}}\right)=\lambda \phi_{\mathcal{G}}\left(s, x_{0}, \sigma_{\mathcal{G}}\right)$ for all $s \geq 0$, by (10). Let us fix $\tau>0$, and consider the ball $\mathbb{B}(0, \bar{R}(\tau))$, with $\bar{R}(\tau)>0$ small enough in order to satisfy

$$
\theta_{i}\left(\tau_{\mathcal{G}}^{\star}, x\right) \geq \tau, \quad \forall|x| \leq \bar{R}(\tau), \forall i \in \mathcal{I} .
$$

Again, this is possible by recalling property (15). Consider any sequence $\left\{\lambda_{\ell}\right\}_{\ell \in \mathbb{N}}, 0<\lambda_{\ell}<\frac{\bar{R}(\tau)}{\left|x_{0}\right|}$ such that $\lambda_{\ell} \rightarrow 0$ as $\ell \rightarrow \infty$. Define the sequence $x_{0 \ell}:=\lambda_{\ell} x_{0} \rightarrow 0$ and

$$
S_{\ell}:=\inf \left\{s \in \mathbb{R}_{+}|| \phi_{\mathcal{G}}\left(s, x_{0 \ell}, \sigma_{\mathcal{G}}\right) \mid \geq \bar{R}(\tau)\right\},
$$

i.e. the first time at which the solution starting at $x_{0 \ell}$ w.r.t. $\sigma_{\mathcal{G}}$ is outside the ball $\mathbb{B}(0, \bar{R}(\tau))$. For all $\ell \in \mathbb{N}, S_{\ell}$ is finite, since $\phi_{\mathcal{G}}\left(\cdot, x_{0 \ell}, \sigma_{\mathcal{G}}\right)$ is a diverging solution. For any $\ell \in \mathbb{N}$, consider the switching signal $\sigma_{\ell} \in \mathcal{S}_{\mathrm{dw}}(\tau)$, and the function $\chi_{\ell}: \mathbb{R}_{+} \rightarrow \mathbb{R}_{+}$, given by Lemma 4 , such that

$$
\phi_{\mathcal{G}}\left(s, x_{0 \ell}, \sigma_{\mathcal{G}}\right)=\phi_{\mathcal{F}}\left(\chi_{\ell}(s), x_{0 \ell}, \sigma_{\ell}\right), \quad \forall s \in \mathbb{R}_{+} .
$$

Defining $t_{\ell}:=\chi_{\ell}\left(S_{\ell}\right)$ we now prove the statement in item 2. For any switching instant $t_{j}^{\sigma_{\ell}}<t_{\ell}$, let us define $x_{j}^{\ell}:=\phi_{\mathcal{F}}\left(t_{j}^{\sigma_{\ell}}, x_{0 \ell}, \sigma_{\ell}\right)$. By definition of $\bar{R}(\tau)$ in (23) and using (36) in Appendix A.2, we have

$$
t_{j+1}^{\sigma_{\ell}}-t_{j}^{\sigma_{\ell}}=\theta_{\sigma_{\mathcal{G}}\left(t_{j}^{\sigma_{\mathcal{G}}}\right)}\left(t_{j+1}^{\sigma_{\mathcal{G}}}-t_{j}^{\sigma_{\mathcal{G}}}, x_{j}^{\ell}\right) \geq \theta_{\sigma_{\mathcal{G}}\left(t_{j}^{\sigma_{\mathcal{G}}}\right)}\left(\tau_{\mathcal{G}}^{\star}, x_{j}^{\ell}\right) \geq \tau
$$

since $\left|x_{j}^{\ell}\right|<r(\tau)$, and thus $\sigma_{\ell} \in \mathcal{S}_{\mathrm{dw}}(\tau)$ (at least until $t_{\ell}$ ), for any $\ell \in \mathbb{N}$. Recalling (17) we have

$$
\phi_{\mathcal{F}}\left(t_{\ell}, \lambda_{\ell} x_{0}, \sigma_{\ell}\right)=\phi_{\mathcal{G}}\left(S_{\ell}, \lambda_{\ell} x_{0}, \sigma_{\mathcal{G}}\right) \notin \mathbb{B}(0, \bar{R}(\tau)),
$$

by definition of $S_{\ell}$, thus proving item 2 .

Remark 3. Rephrasing our result, in proof of item 1 of Theorem 1 we have proved that, fixing a $\tau>0$, for any $x_{0} \in \mathbb{R}^{n}$ and any $\sigma \in \mathcal{S}_{\mathrm{dw}}(\tau)$ there exists a finite time $T\left(x_{0}, \sigma\right) \leq 0$ such that $\left|\phi_{\mathcal{F}}\left(t, x_{0}, \sigma\right)\right| \leq R(\tau)$, $\forall t \geq T\left(x_{0}, \sigma\right)$. Considering the "worst" switching signal and using Lemma 2 , it is possible to provide a finite upper bound $T>0$ such that $T\left(x_{0}, \sigma\right) \leq T$, for any $x_{0} \in \mathbb{R}^{n}$ and any $\sigma \in \mathcal{S}_{\mathrm{dw}}(\tau)$. An estimation of this bound is provided in [1, Theorem 3], where a construction relying on converse Lyapunov theorems for homogeneous systems is proposed. 

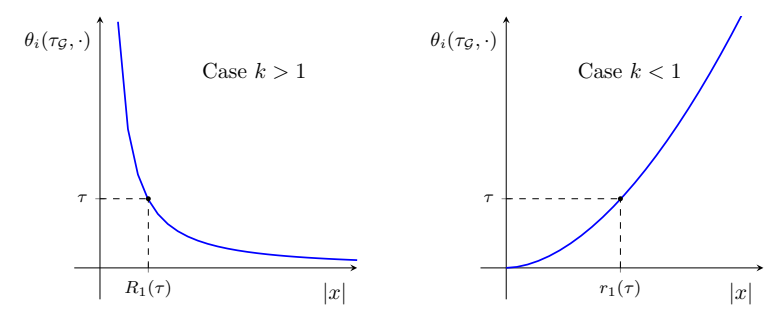

(a) Construction of $R_{1}(\tau)(\mathrm{b})$ Construction of $r_{1}(\tau)$ in (19). in (26).

Figure 2: Qualitative behavior of the functions $\theta_{i}: \mathbb{R}_{\geq 0} \times \mathbb{R}^{n} \rightarrow \mathbb{R}_{\geq 0}$ with respect to the second argument.

Remark 4. The "negative" part of Theorem 1, i.e. Item 2, requires, as hypothesis, the existence of diverging solution of the reduced switched system $\dot{x}(t)=g_{\sigma(t)}(x(t))$, for some $\tau>0$ and $\sigma \in \mathcal{S}_{\mathrm{dw}}(\tau)$. This problem, i.e. the existence of diverging solutions, is non-trivial even for switching systems with linear sub-dynamics, see [8]. Recent works ([7] and [5, Section 2.2]) discuss some aspects of computing Lyapunov exponent and its relation with boundedness of solutions of dwell-time switched systems.

The crucial property used in the proof of Theorem 1, is the fact that, if $k>1$, for a fixed $\tau>0$, the function

$$
\begin{aligned}
& \theta_{i}(\tau, \cdot): \mathbb{R}^{n} \backslash\{0\} \rightarrow \mathbb{R} \quad \text { is radially decreasing, and } \\
& \forall \widehat{x} \in \mathbb{S}_{n-1}, \lim _{\lambda \rightarrow+\infty} \theta_{i}(\tau, \lambda \widehat{x})=0, \lim _{\lambda \rightarrow 0^{+}} \theta_{i}(\tau, \lambda \widehat{x})=+\infty,
\end{aligned}
$$

for each $i \in \mathcal{I}$, recalling (15). In the case $k<1, k \neq 0$, these properties are somehow inverted: fixing any $\tau>0$, we have

$$
\begin{aligned}
& \theta_{i}(\tau, \cdot): \mathbb{R}^{n} \backslash\{0\} \rightarrow \mathbb{R} \text { is radially increasing, and } \\
& \forall \widehat{x} \in \mathbb{S}_{n-1}, \lim _{\lambda \rightarrow+\infty} \theta_{i}(\tau, \lambda \widehat{x})=+\infty, \lim _{\lambda \rightarrow 0^{+}} \theta_{i}(\tau, \lambda \widehat{x})=0,
\end{aligned}
$$

for each $i \in \mathcal{I}$, again by (15). In the case $(k<1)$, we extend by continuity the functions $\theta_{i}(\tau, \cdot)$ to the whole $\mathbb{R}^{n}$, defining $\theta_{i}(\tau, 0)=0$ for any $i \in \mathcal{I}, \tau>0$. Noting this change in the asymptotic properties of the "time-translation" functions, we obtain the following result.

Theorem 2 (Case $k<1)$. Consider a finite family of vector fields satisfying Assumption $1, \mathcal{F}=\left\{f_{i}\right\}_{i \in \mathcal{I}} \subset$ $\mathcal{H}_{n}^{k}$, with $k<1, k \neq 0$ and suppose that the subsystem $\dot{x}=f_{i}(x)$ is GAS, for each $i \in \mathcal{I}$. Consider the set $\mathcal{G}=\left\{g_{i}\right\}_{i \in \mathcal{I}} \subset \mathcal{H}_{n}^{1}$ obtained by the reductions of degree 1 of the $f_{i}$. Then:

1' (Local Asymptotic Stability) For every $\tau>0$, there exists an $r(\tau)>0$ such that the origin is a uniform (local) asymptotically stable equilibrium in $\mathbb{B}(0, r(\tau))$ of $(1)$ on $\mathcal{S}_{\mathrm{dw}}(\tau)$; i.e. the property in Definition 1 holds only when considering $\left|x_{0}\right|<r(\tau)$.

2' (Diverging Solutions) Suppose that there exist $\tau_{\mathcal{G}}^{\star}>0, \sigma_{\mathcal{G}} \in \mathcal{S}_{\mathrm{dw}}\left(\tau_{\mathcal{G}}^{\star}\right)$ and $x_{0} \in \mathbb{R}^{n}$ such that the solution of the switched system $\dot{x}=g_{\sigma_{\mathcal{G}}(t)}(x)$ denoted by $\phi_{\mathcal{G}}\left(\cdot, x_{0}, \sigma_{\mathcal{G}}\right): \mathbb{R}_{\geq 0} \rightarrow \mathbb{R}^{n}$ is diverging, i.e.

$$
\limsup _{s \rightarrow+\infty}\left|\phi_{\mathcal{G}}\left(s, x_{0}, \sigma_{\mathcal{G}}\right)\right|=+\infty .
$$

Then, for every $\tau>0$, there exists $z_{0} \in \mathbb{R}^{n}$ and $\sigma \in \mathcal{S}_{\mathrm{dw}}(\tau)$ such that $\lim \sup _{t \rightarrow \infty}\left|\phi_{\mathcal{F}}\left(t, z_{0}, \sigma\right)\right|=+\infty$.

Since this proof is obtained as a "dual" version of the proof of Theorem 1, some details are omitted.

Proof. Again, we will denote by $\theta_{i}: \mathbb{R} \times \mathbb{R}^{n} \rightarrow \mathbb{R}$ and $\phi_{g_{i}}: \mathbb{R} \times \mathbb{R}^{n} \rightarrow \mathbb{R}^{n}$, the maps defined by (13) and (12), respectively, when considering the sub-vector field $f_{i}: \mathbb{R}^{n} \rightarrow \mathbb{R}^{n}$. Recalling Lemma 1 , there exists a $\tau_{\mathcal{G}}>0$, such that (18) is GAS on $\mathcal{S}_{\mathrm{dw}}\left(\tau_{\mathcal{G}}\right) ; \beta_{\mathcal{G}} \in \mathcal{K} \mathcal{L}$ denotes the function characterizing the GAS property, as in 
Definition 1.

- Let us prove item 1': consider any $\tau>0$, take $r_{1}(\tau)>0$ small enough such that

$$
\theta_{i}\left(\tau_{\mathcal{G}}, x\right) \leq \tau, \quad \forall|x| \leq r_{1}(\tau), \forall i \in \mathcal{I}
$$

which is possible due to (25), see also Figure 2b. Using this relation, we can prove item 1' using the following lemma:

Lemma 6. For a given $\tau>0$, consider $r_{1}(\tau)>0$ that satisfies (26). If $r(\tau)>0$ is chosen small enough such that

$$
\beta_{\mathcal{G}}(0, r(\tau)) \leq r_{1}(\tau)
$$

then item 1' holds, considering the ball $\mathbb{B}(0, r(\tau))$.

Proof of Lemma 6. Take any $x_{0} \in \mathbb{B}(0, r(\tau))$ and any $\sigma \in \mathcal{S}_{\mathrm{dw}}(\tau)$. The solution $\phi_{\mathcal{F}}\left(\cdot, x_{0}, \sigma\right): \mathbb{R}_{\geq 0} \rightarrow \mathbb{R}^{n}$ corresponds, after re-scaling the time-axis, to a solution $\phi_{\mathcal{G}}\left(\cdot, x_{0}, \sigma_{\mathcal{G}}\right): \mathbb{R}_{\geq 0} \rightarrow \mathbb{R}^{n}$ of system (18) for some $\sigma_{\mathcal{G}} \in \mathcal{S}$. More precisely, define $T:=\inf \left\{t \geq 0 \mid \phi_{\mathcal{F}}\left(t, x_{0}, \sigma\right)=0\right\}$. This $T>0$ could be finite, since, recalling Lemma 2, subsystems $\dot{x}=f_{i}(x)$ are finite-time stable. Then, as stated in (16) in Lemma 4, there exist a continuous increasing function $\gamma:[0, T) \rightarrow \mathbb{R}_{\geq 0}$ and a $\sigma_{\mathcal{G}} \in \mathcal{S}$ such that $\phi_{\mathcal{F}}\left(t, x_{0}, \sigma\right)=\phi_{\mathcal{G}}\left(\gamma(t), x_{0}, \sigma_{\mathcal{G}}\right)$, $\forall t \in[0, T)$. Since $\left|x_{0}\right|<r(\tau)$, recalling (26), (27) and (35), it can be iteratively shown that $\sigma_{\mathcal{G}} \in \mathcal{S}_{\mathrm{dw}}\left(\tau_{\mathcal{G}}\right)$ and the solution $\phi_{\mathcal{F}}\left(\cdot, x_{0}, \sigma\right)=\phi_{\mathcal{G}}\left(\gamma(\cdot), x_{0}, \sigma_{\mathcal{G}}\right):[0, T) \rightarrow \mathbb{R}^{n}$ does not leave $\overline{\mathbb{B}}\left(0, r_{1}(\tau)\right)$. Thus,

$$
\begin{array}{rlrl}
\left|\phi_{\mathcal{F}}\left(t, x_{0}, \sigma\right)\right| \leq \beta_{\mathcal{G}}\left(\gamma(t), x_{0}\right), & & \forall t \in[0, T) \\
\left|\phi_{\mathcal{F}}\left(t, x_{0}, \sigma\right)\right| & =0, & & \forall t \in[T,+\infty)
\end{array}
$$

concluding the proof of Lemma 6 .

- We now prove item 2'. Consider $\sigma_{\mathcal{G}} \in \mathcal{S}_{\mathrm{dw}}\left(\tau_{\mathcal{G}}^{\star}\right)$ for some $\tau_{\mathcal{G}}^{\star}>0$, and $x_{0}$ such that the corresponding solution of system (18) is diverging. For a fixed $\tau>0$, consider a scalar $\underline{r}(\tau)>0$ such that

$$
\theta_{i}\left(\tau_{\mathcal{G}}^{\star}, x\right)>\tau, \quad \forall|x| \geq \underline{r}(\tau), \quad \forall i \in \mathcal{I},
$$

which, again, is possible by (25). Let us now consider $\lambda>0$ large enough, such that $x_{j}:=\phi_{\mathcal{G}}\left(t_{j}^{\sigma_{\mathcal{G}}}, \lambda x_{0}, \sigma_{\mathcal{G}}\right) \notin$ $\mathbb{B}(0, \underline{r}(\tau))$ for all switching instant $t_{j}^{\sigma_{\mathcal{G}}} \geq 0$. This is possible since, by definition of diverging solution, we have that $\inf \left\{\left|\phi_{\mathcal{G}}\left(t_{j}^{\sigma_{\mathcal{G}}}, x_{0}, \sigma_{\mathcal{G}}\right)\right| \mid t_{j}^{\sigma_{\mathcal{G}}} \geq 0\right\}>0$, and recalling that $\phi_{\mathcal{G}}\left(s, \lambda x_{0}, \sigma_{\mathcal{G}}\right)=\lambda \phi_{\mathcal{G}}\left(s, x_{0}, \sigma_{\mathcal{G}}\right)$, for any $s \in \mathbb{R}_{\geq 0}$ and any $\lambda>0$. By Lemma 4 , there exist $\sigma \in \mathcal{S}$, and a continuous increasing function $\chi: \mathbb{R}_{\geq 0} \rightarrow \mathbb{R}_{\geq 0}$ with $\chi(0)=0$ such that

$$
\phi_{\mathcal{F}}\left(\chi(s), \lambda x_{0}, \sigma\right)=\phi_{\mathcal{G}}\left(s, \lambda x_{0}, \sigma_{\mathcal{G}}\right) \forall s \in \mathbb{R}_{\geq 0} .
$$

Since $\phi_{\mathcal{G}}\left(\cdot, \lambda x_{0}, \sigma_{\mathcal{G}}\right)$ is diverging and solution of $(1)$ are forward complete, it is clear that $\lim _{s \rightarrow \infty} \chi(s)=+\infty$, see the reasoning in Lemma 4 . Recalling that $x_{j} \notin \mathbb{B}(0, \underline{r}(\tau))$, for any $j \in \mathbb{N}$, by (28) and (36) we have that $\sigma \in \mathcal{S}_{\mathrm{dw}}(\tau)$. We concluding noting that $\lim \sup _{s \rightarrow+\infty} \phi_{\mathcal{F}}\left(\chi(s), \lambda x_{0}, \sigma\right)=\lim \sup _{s \rightarrow+\infty} \phi_{\mathcal{G}}\left(s, \lambda x_{0}, \sigma_{\mathcal{G}}\right)=$ $+\infty$

Remark 5. In Theorem 1 and Theorem 2 we provided, for any dwell-time threshold $\tau>0$, some "partial" stability properties, i.e. ultimate boundlessness in the case $k>1$ (item 1 of Theorem 1), and local stability in the case $k<1$ (item 1' of Theorem 2). We showed how the attracting set and the region of attraction, respectively, depend on the chosen $\tau>0$. On the other hand, avoiding the trivial case of systems asymptotically stable under arbitrary switching signals, we proved how these properties cannot be extended on the whole state space, proving instability in the case $k>1$ (item 2 of Theorem 1) and the existence of unbounded solutions in the case $k<1$ (item 2' of Theorem 2). We have thus proven how the fact that, for a family of GAS subsystems, there always exists a (large enough) dwell time for which the switched system (1) is globally asymptotically stable (proven in [19, Lemma 2] for the linear case) cannot be, in general, extended to the non-linear case. Moreover, in Theorem 1 and Theorem 2, the common idea is to fix a dwell time $\tau>0$, and then study the (in-)stability properties of (1) with respect to some sets. The following corollary 
provides the statements in other direction: fixing a neighborhood of the origin (for $k>1$ ) or a neighborhood of infinity (for $k<1$ ), it is possible to select a $\tau>0$ big enough such that stability/convergence properties hold in an appropriate sense with respect to the chosen sets.

Corollary 1. Consider a finite family of vector fields $\mathcal{F}=\left\{f_{i}\right\}_{i \in \mathcal{I}} \subset \mathcal{H}_{n}^{k}$ satisfying Assumption 1 and suppose that each subsystem $\dot{x}=f_{i}(x)$ is $G A S, i \in \mathcal{I}$. Then:

A) If $k>1$, then, $\forall \varepsilon>0$, there exists $\tau(\varepsilon)>0$ such that

$$
\limsup _{t \rightarrow \infty}\left|\phi_{\mathcal{F}}\left(t, x_{0}, \sigma\right)\right| \leq \varepsilon, \forall x_{0} \in \mathbb{R}^{n}, \forall \sigma \in \mathcal{S}_{\mathrm{dw}}(\tau(\varepsilon)),
$$

or, in other words, $\overline{\mathbb{B}}(0, \varepsilon)$ is a compact attractor of $(1)$ on $\mathcal{S}_{\mathrm{dw}}(\tau(\varepsilon))$.

$B)$ If $k<1, k \neq 0$, then, $\forall M>0$, there exists $\tau(M)>0$ such that

$$
\lim _{t \rightarrow \infty} \phi_{\mathcal{F}}\left(t, x_{0}, \sigma\right)=0, \forall x_{0} \in \mathbb{B}(0, M), \forall \sigma \in \mathcal{S}_{\mathrm{dw}}(\tau(M)),
$$

or, in other words, $\mathbb{B}(0, M)$ is contained in the region of attraction of $(1)$ on $\mathcal{S}_{\mathrm{dw}}(\tau(M))$.

Proof. Denote again by $g_{i}: \mathbb{R}^{n} \rightarrow \mathbb{R}^{n}, \phi_{g_{i}}: \mathbb{R} \times \mathbb{R}^{n} \rightarrow \mathbb{R}^{n}$ and $\theta_{i}: \mathbb{R} \times \mathbb{R}^{n} \rightarrow \mathbb{R}$ the maps defined by (8), (13) and (12) respectively, when considering the sub-vector field $f_{i}: \mathbb{R}^{n} \rightarrow \mathbb{R}^{n}$. With $\beta_{i} \in \mathcal{K} \mathcal{L}$, we denote the function characterizing the GAS property of the system $\dot{x}=f_{i}(x)$, for any $i \in \mathcal{I}$. Fix $\tau_{\mathcal{G}}>0$, such that (18) is UGAS on $\mathcal{S}_{\mathrm{dw}}\left(\tau_{\mathcal{G}}\right) ; \beta_{\mathcal{G}} \in \mathcal{K} \mathcal{L}$ denotes the function characterizing the UGAS property, as in Definition 1. For proving item A), consider $r(\varepsilon):=\max _{i \in \mathcal{I}} \beta_{i}(0, \varepsilon)$ and define $\tau(\varepsilon)>0$ big enough such that $\theta_{i}\left(\tau_{\mathcal{G}}, x\right) \leq \tau(\varepsilon)$, $\forall|x| \geq r(\varepsilon), \forall i \in \mathcal{I}$ which is possible due to the properties stated in (24). The statement then follows by Lemma 5 .

For proving item B), consider $R(M):=\max _{i \in \mathcal{I}} \beta_{i}(0, M)$, and consider $\tau(M)>0$ big enough such that $\theta_{i}\left(\tau_{\mathcal{G}}, x\right) \leq \tau(M), \forall|x| \leq R(M), \forall i \in \mathcal{I}$, which is again possible due to (25). Using Lemma 6 , the solutions starting in $\mathbb{B}(0, M)$ therefore converge to zero.

Concluding this section, we note that our results (Theorem 1, Theorem 2 and Corollary 1) can be generalized in the case of homogeneous vector fields $\left\{f_{i}\right\}_{i \in \mathcal{I}}$ with mode-dependent degrees $k_{i} \in \mathbb{R}, i \in \mathcal{I}$, with the same "limiting behavior", i.e. $k_{i}>1\left(\right.$ or $\left.k_{i}<1\right)$, for all $i \in \mathcal{I}$.

\section{Beyond Homogeneity: General Case}

In Section 4, we analyzed stability/boundedness properties of switching systems composed of homogeneous subsystems. The crucial hypothesis in the proofs of previous results was the "extremal" nature of the subsystems, i.e. the behavior of $\lim _{|x| \rightarrow 0^{+}}\left|f_{i}(x)\right|$, and $\lim _{|x| \rightarrow+\infty}\left|f_{i}(x)\right|$. Thus, it seems reasonable that, regardless of the specific structure of the subsystems, when these critical regions (neighborhoods of origin/infinity) are disregarded, the stability properties are somehow conserved by the switched system (1), under (sufficiently large) dwell-time policy. This intuition is formalized in the following semi-global and practical result.

Proposition 2 (Semi-global \& practical dwell-time stability). Consider a finite family of vector fields $\mathcal{F}=$ $\left\{f_{i}\right\}_{i \in \mathcal{I}} \subset \mathcal{C}^{1}\left(\mathbb{R}^{n} \backslash\{0\}, \mathbb{R}^{n}\right)$ satisfying Assumption 1, and suppose that $\dot{x}=f_{i}(x)$ is $G A S$, for each $i \in \mathcal{I}$. For every $M>\varepsilon>0$ there exists a $\tau=\tau(\varepsilon, M)>0$ such that

$$
\limsup _{t \rightarrow+\infty}\left|\phi_{\mathcal{F}}\left(t, x_{0}, \sigma\right)\right| \leq \varepsilon, \forall\left|x_{0}\right| \leq M, \forall \sigma \in \mathcal{S}_{\mathrm{dw}}(\tau) .
$$

Similarly, for every $\tau>0$ there exist $\varepsilon=\varepsilon(\tau)>0$ and $M=M(\tau)>0$ for which (29) holds.

Proof. Let us consider functions $\beta_{i} \in \mathcal{K} \mathcal{L}$ characterizing the GAS property of the subsystems $\dot{x}=f_{i}(x)$, for each $i \in \mathcal{I}$, respectively. Consider $M>\varepsilon>0$, and select $0<\varepsilon_{2} \leq \varepsilon_{1} \leq \varepsilon$ such that

$$
\beta_{i}\left(0, \varepsilon_{1}\right) \leq \varepsilon, \quad \forall i \in \mathcal{I}, \text { and } \beta_{i}\left(0, \varepsilon_{2}\right) \leq \varepsilon_{1}, \quad \forall i \in \mathcal{I}
$$


(this is possible by finiteness of $\mathcal{I}$ and by definition of $\mathcal{K} \mathcal{L}$-functions). Fix any $0<\xi<1$, it now suffices to consider a $\tau>0$ large enough such that

$$
\beta_{i}(\tau, s) \leq(1-\xi) s, \quad \forall s \in\left[\varepsilon_{2}, M\right], \forall i \in \mathcal{I} .
$$

Again this choice is possible by finiteness of $\mathcal{I}$ and by compactness of the interval $\left[\varepsilon_{2}, M\right] \subset \mathbb{R}_{\geq 0} \backslash\{0\}$. Let us now prove that (29) holds: consider $x_{0} \in \overline{\mathbb{B}}(0, M)$ and $\sigma \in \mathcal{S}_{\mathrm{dw}}(\tau)$. Without loss of generality, we can suppose that $\sigma$ has an infinite number of discontinuities since for eventually constant signals condition (29) trivially holds. Denote the set of switching instants by $\left\{t_{j}^{\sigma}\right\}_{j \in \mathbb{N}}$. Let us call $x_{j}:=\phi_{\mathcal{F}}\left(t_{j}^{\sigma}, x_{0}, \sigma\right), j \in \mathbb{N}$, i.e. the values of the solution at switching instants, and let $\bar{j}:=\inf \left\{j \in \mathbb{N}|| x_{j} \mid \leq \varepsilon_{2}\right\}$ (possibly $\bar{j}=0$ ). Using (31), for all $j \leq \bar{j}$, we get $\left|x_{j}\right| \leq(1-\xi)^{j}\left|x_{0}\right| \leq(1-\xi)^{j} M$ and since $(1-\xi)^{j} \rightarrow 0$ as $j \rightarrow \infty$, it follows that $\bar{j}$ is finite. From (30), we now have $\left|x\left(t, x_{0}, \sigma\right)\right| \leq \varepsilon, \forall t \geq t \frac{\sigma}{j}$. Since $x_{0} \in \overline{\mathbb{B}}(0, M)$ is arbitrary, and $\sigma \in \mathcal{S}_{\mathrm{dw}}(\tau)$, then $(29)$ holds.

Next, for a fixed $\tau>0$, we construct $0<\varepsilon(\tau)<M(\tau)$ for which (29) holds, simply following the ideas already presented. Take $0<\xi<1$, and chose $\varepsilon_{2}>0$ and $M>0$ such that (31) holds. Then define $\varepsilon_{1}, \varepsilon>0$, such that $0<\varepsilon_{1} \leq \varepsilon$ and (30) holds. We can now conclude, following the reasoning we have already developed, that (29) holds.

Interestingly, an alternative proof of Proposition 2 can be obtained relying on a Lyapunov converse argument, using the ideas sketched in [23, Section 4.1]. Despite this general result, proof techniques based on reduction of the degree used in Section 4 (see Lemma 3), seem unfeasible in a generic setting. However, the proposed method can be adapted for the particular (but remarkable) case of positive scalar multiplications of linear systems.

Proposition 3 (Scalar multiples of linear subsystems). Consider $\mathcal{A}:=\left\{A_{i}\right\}_{i \in \mathcal{I}} \subset \mathbb{R}^{n \times n}$ such that $A_{i}$ is Hurwitz, $\forall i \in \mathcal{I}$. Consider, for any $i \in \mathcal{I}$ a function $\varphi_{i}: \mathbb{R}_{\geq 0} \rightarrow \mathbb{R}$ such that $\varphi_{i}(0)=0, \varphi_{i}(s)>0$ if $s \neq 0$ and $\varphi_{i} \in \mathcal{C}^{1}\left(\mathbb{R}_{\geq 0} \backslash\{0\}, \mathbb{R}\right)$. Consider the subsystems defined by $f_{i}(x):=\varphi_{i}(|x|) A_{i} x$ and the switched system

$$
\dot{x}=f_{\sigma(t)}(x) .
$$

1. If there exists $K_{\infty}>0$ such that

$$
\liminf _{s \rightarrow+\infty} \varphi_{i}(s) \geq K_{\infty}, \quad \forall i \in \mathcal{I},
$$

then for some $\tau>0,(32)$ is uniformly ultimately bounded on $\mathcal{S}_{\mathrm{dw}}(\tau)$ (cf. item 1 of Theorem 1).

2. If there exists $k_{0}>0$ such that

$$
\liminf _{s \rightarrow 0^{+}} \varphi_{i}(s) \geq k_{0}, \quad \forall i \in \mathcal{I},
$$

then for some $\tau>0,(32)$ is uniformly (locally) asymptotically stable on $\mathcal{S}_{\mathrm{dw}}(\tau)$ (cf. item 1' of Theorem 2).

Proof. Recalling Lemma 1, let us fix $\tau_{\mathcal{A}}>0$ as the dwell-time (possibly minimal) for which the switched system $\dot{x}=A_{\sigma(t)} x$ is UGAS on $\mathcal{S}_{\mathrm{dw}}\left(\tau_{\mathcal{A}}\right)$. Denoting by $\phi_{i}: \mathbb{R}_{\geq 0} \times \mathbb{R}^{n} \rightarrow \mathbb{R}^{n}$ the flow-maps of the subsystems $\dot{x}=f_{i}(x)$ and reasoning as in Lemma 3 , it can be shown that $e^{A_{i} t} x=\phi_{i}\left(\theta_{i}(t, x), x\right), \forall t \in \mathbb{R}_{\geq 0}, \forall x \in \mathbb{R}^{n}$, $\forall i \in \mathcal{I}$, where the functions $\theta_{i}: \mathbb{R}_{\geq 0} \times \mathbb{R}^{n} \rightarrow \mathbb{R}$ are defined by

$$
\theta_{i}(t, x):=\int_{0}^{t} \frac{1}{\varphi_{i}\left(\left|e^{A_{i} s} x\right|\right)} d s .
$$

Case 1: Fix a $0<K<K_{\infty}$, by (33), there exists $\eta_{\infty}>0$ such that $\varphi_{i}(s)>K, \forall s>\eta_{\infty}, \forall i \in \mathcal{I}$. Consider $R_{1} \geq \eta_{\infty}$ such that $\left|e^{A_{i} t} x\right|>\eta_{\infty}, \forall|x| \geq R_{1}, \forall t \in\left[0, \tau_{\mathcal{A}}\right]$ and $\forall i \in \mathcal{I}$. Considering any $x \in \mathbb{R}^{n},|x| \geq R_{1}$, we thus have

$$
\theta_{i}\left(\tau_{\mathcal{A}}, x\right):=\int_{0}^{\tau_{\mathcal{A}}} \frac{1}{\varphi_{i}\left(\left|e^{A_{i} s} x\right|\right)} d s \leq \int_{0}^{\tau_{\mathcal{A}}} \frac{1}{K} d s=\frac{\tau_{\mathcal{A}}}{K} .
$$


We can now choose $\tau>0$ large enough such that $\tau>\frac{\tau_{\mathcal{A}}}{K}$, concluding that $\theta_{i}\left(\tau_{\mathcal{A}}, x\right) \leq \tau, \forall|x| \geq R_{1}, \forall i \in \mathcal{I}$. We can now conclude the ultimate boundedness by applying Lemma 5 , mutadis mutandis.

Case 2: Fix a $0<k<k_{0}$, by (34) there exists $\eta_{0}>0$ such that $\varphi_{i}(s)>k, \forall 0<s<\eta_{0}, \forall i \in \mathcal{I}$. Consider $r_{1}>0$ such that $\left|e^{A_{i} t} x\right|<\eta_{0}, \forall|x| \leq r_{1}, \forall t \in\left[0, \tau_{\mathcal{A}}\right]$ and $\forall i \in \mathcal{I}$. Now take any $x \in \mathbb{R}^{n}$ such that $|x| \leq r_{1}$ and any $i \in \mathcal{I}$, computing we obtain

$$
\theta_{i}\left(\tau_{\mathcal{A}}, x\right)=\int_{0}^{\tau_{\mathcal{A}}} \frac{1}{\varphi_{i}\left(\left|e^{A_{i} s} x\right|\right)} d s \leq \int_{0}^{\tau_{\mathcal{A}}} \frac{1}{k} d s=\frac{\tau_{\mathcal{A}}}{k} .
$$

Let choose $\tau>0$ big enough such that $\tau>\frac{\tau_{\mathcal{A}}}{k}$, implying $\theta_{i}\left(\tau_{\mathcal{A}}, x\right) \leq \tau, \forall|x| \leq r_{1}, \forall i \in \mathcal{I}$. The arguments for local asymptotic stability follow from the proof of Lemma 6 .

\section{Conclusion}

We studied stability of switched nonlinear systems under dwell-time constraints. Differently from the linear subsystems case, the stability of each subsystem does not imply, in general, the existence of a large enough dwell time which stabilizes the switched system. Nonetheless, we provided new results, showing how weaker stability/asymptotic properties could be proved, depending on the behavior of the subsystems close and/or far from the origin. The statements are first developed for homogeneous subsystems, and then some generalizations in a generic framework are provided. As possible future research path, we plan to use the proposed results to designing switching signals for practical stability (for the case of degree $k>1$ ) or local stability $(k<1)$ of homogeneous switched systems.

\section{A Appendix}

In this Appendix we prove Proposition 1 and Lemma 4..

\section{A.1 Proof of Proposition 1}

Proof. Item 1: The continuity of $\theta(\cdot, x): \mathbb{R}_{\geq 0} \rightarrow \mathbb{R}$ for each $x \in \mathbb{R}^{n} \backslash\{0\}$ follows directly from its definition in (12). The continuity with respect to second argument follows by the fact that $f \in \mathcal{C}^{1}\left(\mathbb{R}^{n} \backslash\{0\}, \mathbb{R}^{n}\right)$ and, by continuous dependence on initial conditions, $\phi_{g}(t, \cdot): \mathbb{R}^{n} \rightarrow \mathbb{R}^{n}$ is continuous, see for example $[13$, Theorem 3.5].

Item 2: Exploiting the relation (10) and the homogeneity of $f$ and $g_{f}$, we have, for every $x \in \mathbb{R}^{n} \backslash\{0\}$ and $t \geq 0$, and each $\lambda>0$,

$$
\begin{aligned}
\theta(t, \lambda x) & =\int_{0}^{t} \frac{\left|f\left(\phi_{g}(s, \lambda x)\right)\right|^{\frac{1}{k}}}{\mid f\left(\phi_{g}(s, \lambda x) \mid\right.} d s=\int_{0}^{t} \frac{\left|f\left(\phi_{g}(s, \lambda x)\right)\right|^{\frac{1}{k}}}{\left|f\left(\lambda \phi_{g}(s, x)\right)\right|} d s \\
& =\int_{0}^{t} \frac{\lambda\left|f\left(\phi_{g}(s, x)\right)\right|^{\frac{1}{k}}}{\lambda^{k}\left|f\left(\phi_{g}(s, x)\right)\right|} d s \\
& =\frac{1}{\lambda^{k-1}} \int_{0}^{t} \frac{\left|f\left(\phi_{g}(s, x)\right)\right|^{\frac{1}{k}}}{\left|f\left(\phi_{g}(s, x)\right)\right|} d s=\frac{1}{\lambda^{k-1}} \theta(t, x),
\end{aligned}
$$

so that (15) holds.

Item 3: Due to the uniqueness of solutions (obtained from Assumption 1), we note that $\phi_{g}(t, x) \neq 0$ for all $x \neq 0$, for all $t \in \mathbb{R}_{\geq 0}$. This yields $\frac{\left|f\left(\phi_{g}(t, x)\right)\right| \frac{1}{k}}{\left|f\left(\phi_{g}(t, x)\right)\right|}>0$ for all $t \in \mathbb{R}_{\geq 0}$, and the desired statement follows from (12).

Item 4: Straightforward, since $g_{f}=f$ in the case $k=1$, thus $\theta(t, x)=\int_{0}^{t} 1 d s$, for any $x \in \mathbb{R}^{n} \backslash\{0\}$.

Items 4'. and 4". Given any $x \in \mathbb{R}^{n} \backslash\{0\}$, the behavior of $\theta(t, x)$ in the cases $(k<1)$ and $(k>1)$ as $t$ goes to infinity follows from (13) and Lemma 2. 


\section{A.2 Proof of Lemma 4}

Proof. Consider $x_{0} \neq 0, \sigma \in \mathcal{S}$ and the associated set of switching instant $\left\{t_{j}^{\sigma}\right\}$. We proceed by induction on $j$ in defining $\sigma_{\mathcal{G}}$ and $\gamma$ as in (16). Firstly, by convention, recall that $t_{0}^{\sigma}=t_{0}^{\sigma_{\mathcal{G}}}=0$; and let $i_{0}:=\sigma(0) \in \mathcal{I}$. We proceed by a recursion argument.

Case $j=0$ : We consider two scenarios depending upon when $t_{1}^{\sigma}$ occurs.

- If the first switching instant $t_{1}^{\sigma}$ does not exist (because $\sigma$ "never jumps" ) or $t_{1}^{\sigma} \geq T$, we define

$$
\left\{\begin{array}{l}
\sigma_{\mathcal{G}}(s)=\sigma(0)=i_{0} \quad \forall s \in[0,+\infty) \\
\gamma(t):=\theta_{i}^{-1}\left(t, x_{0}\right), \quad t \in[0, T)
\end{array}\right.
$$

Recalling (13), it is clear that $\phi_{\mathcal{F}}\left(t, x_{0}, \sigma\right)=\phi_{f_{i_{0}}}\left(t, x_{0}\right)=\phi_{g_{i_{0}}}\left(\gamma(t), x_{0}\right)=\phi_{\mathcal{G}}\left(\gamma(t), x_{0}, \sigma_{\mathcal{G}}\right)$, for each $t \in[0, T)$, concluding this (trivial) case.

- If $t_{1}^{\sigma}<T$, define $t_{1}^{\sigma \mathcal{G}}=\theta_{i_{0}}^{-1}\left(t_{1}^{\sigma}, x_{0}\right), \sigma_{\mathcal{G}}(s)=\sigma(0)=i_{0}$, for $s \in\left[t_{0}^{\sigma_{\mathcal{G}}}, t_{1}^{\sigma \mathcal{G}}\right), \gamma_{\left.\left.\right|_{\left[0, t_{1}\right.}\right)}(t)=\theta_{i_{0}}^{-1}\left(t, x_{0}\right)$. Again by (13), for every $t \in\left[0, t_{1}^{\sigma}\right)$, we have, $\phi_{\mathcal{F}}\left(t, x_{0}, \sigma\right)=\phi_{f_{i_{0}}}\left(t, x_{0}\right)=\phi_{g_{i_{0}}}\left(\gamma(t), x_{0}\right)=\phi_{\mathcal{G}}\left(\gamma(t), x_{0}, \sigma_{\mathcal{G}}\right)$.

Recursive Step. Let us suppose that, for some $j>0$ we have already defined $t_{j}^{\sigma_{\mathcal{G}}}>0, \sigma_{\mathcal{G}}(s)$ for every $s \in\left[0, t_{j}^{\sigma \mathcal{G}}\right)$ and $\gamma(t)$ for every $t \in\left[0, t_{j}^{\sigma}\right)$. Call $x_{j}:=\phi_{\mathcal{F}}\left(t_{j}^{\sigma}, x_{0}, \sigma\right) \in \mathbb{R}^{n} \backslash\{0\}$ and $i_{j}=\sigma\left(t_{j}^{\sigma}\right)$.

- If the next switching instant $t_{j+1}^{\sigma}$ does not exist or $t_{j+1}^{\sigma} \geq T$, we define

$$
\left\{\begin{array}{l}
\sigma_{\mathcal{G}}(s)=\sigma(0)=i_{0} \quad \forall s \in\left[t_{j}^{\sigma_{\mathcal{G}}},+\infty\right) \\
\gamma(t):=\theta_{i_{j}}^{-1}\left(t-t_{j}^{\sigma}, x_{j}\right)+t_{j}^{\sigma_{\mathcal{G}}}, \quad \forall t \in\left[t_{j}^{\sigma}, T\right)
\end{array}\right.
$$

Recalling (13), it is clear that $\phi_{\mathcal{F}}\left(t, x_{0}, \sigma\right)=\phi_{f_{i_{j}}}\left(t-t_{j}^{\sigma}, x_{j}\right)=\phi_{g_{i_{j}}}\left(\theta_{i_{j}}^{-1}\left(t-t_{j}^{\sigma}, x_{j}\right), x_{j}\right)=\phi_{g_{i_{j}}}(\gamma(t)-$ $\left.t_{j}^{\sigma_{\mathcal{G}}}, x_{j}\right)=\phi_{\mathcal{G}}\left(\gamma(t), x_{0}, \sigma_{\mathcal{G}}\right)$, for all $t \in\left[t_{j}^{\sigma}, T\right)$, concluding the proof in this case.

- If $t_{j+1}^{\sigma}<T$, define

$$
\begin{aligned}
t_{j+1}^{\sigma_{\mathcal{G}}} & =t_{j}^{\sigma_{\mathcal{G}}}+\theta_{i_{j}}^{-1}\left(t_{j}^{\sigma}-t_{j-1}^{\sigma}, x_{j}\right), \\
\sigma_{\mathcal{G}}(s) & =\sigma(0)=i_{j}, \quad \forall s \in\left[t_{j}^{\sigma_{\mathcal{G}}}, t_{j+1}^{\sigma_{\mathcal{G}}}\right), \\
\gamma_{\left.\right|_{\left[t_{j}^{\sigma}, t_{j+1}^{\sigma}\right)}(t)} & =\theta_{i_{j}}^{-1}\left(t-t_{j}^{\sigma}, x_{j}\right)+t_{j}^{\sigma_{\mathcal{G}}},
\end{aligned}
$$

Again by (13), we have $\phi_{\mathcal{F}}\left(t, x_{0}, \sigma\right)=\phi_{f_{i_{j}}}\left(t-t_{j}^{\sigma}, x_{j}\right)=\phi_{g_{i_{j}}}\left(\gamma(t)-t_{j}^{\sigma \mathcal{G}}, x_{j}\right)=\phi_{\mathcal{G}}\left(\gamma(t), x_{0}, \sigma_{\mathcal{G}}\right)$, for all $t \in\left[t_{j}^{\sigma}, t_{j+1}^{\sigma}\right)$.

Iterating the procedure a countable number of times (while allowing for $\sigma$ not eventually constant and $T=+\infty)$, we get the desired statement.

The statement in the other direction, leading to (17), can be obtained with a completely similar argument which is briefly sketched here. Given $x_{0} \neq 0$ and $\sigma_{\mathcal{G}} \in \mathcal{S}$, by convention $t_{0}^{\sigma_{\mathcal{G}}}=t_{0}^{\sigma}=0$, and call $\sigma(0)=$ $\sigma_{\mathcal{G}}(0)=i_{0} \in \mathcal{I}$. For all $j \geq 0$, suppose $t_{j}^{\sigma}, \sigma\left(t_{j}^{\sigma}\right)$, and $\left.\chi\right|_{\left[0, t_{j}^{\sigma}\right)}$ are already defined. Call $x_{j}=\phi_{\mathcal{G}}\left(t_{j}^{\sigma_{\mathcal{G}}}, x_{0}, \sigma_{\mathcal{G}}\right)$, and $i_{j}=\sigma\left(x_{j}\right)$. Defining

$$
\begin{aligned}
& t_{j+1}^{\sigma}=t_{j}^{\sigma}+\theta_{i_{j}}\left(t_{j+1}^{\sigma_{\mathcal{G}}}-t_{j}^{\sigma_{\mathcal{G}}}, x_{j}\right), \\
& \sigma(t)=\sigma_{\mathcal{G}}\left(t_{j+1}^{\sigma_{\mathcal{G}}}\right), \quad \forall t \in\left[t_{j}^{\sigma}, t_{j+1}^{\sigma}\right)
\end{aligned}
$$

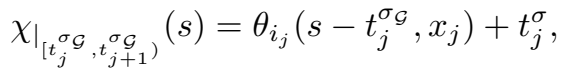

it can be seen that, iterating, one obtains (17). 


\section{References}

[1] A.Y. Aleksandrov, A.A. Kosov, and A.V. Platonov. On the asymptotic stability of switched homogeneous systems. Systems \& Control Letters, 61(1):127 - 133, 2012.

[2] V. Andrieu, L. Praly, and A. Astolfi. Homogeneous approximation, recursive observer design, and output feedback. SIAM Journal on Control and Optimization, 47(4):1814-1850, 2008.

[3] A. Bacciotti and L. Rosier. Liapunov Functions and Stability in Control Theory, volume 267 of Lecture Notes in Control and Information Sciences. Springer-Verlag, 2005.

[4] F. Blanchini and P. Colaneri. Vertex/plane characterization of the dwell-time property for switching linear systems. In 49th IEEE Conference on Decision and Control (CDC), pages 3258-3263, 2010.

[5] F. Boarotto and M. Sigalotti. Dwell-time control sets and applications to the stability analysis of linear switched systems. Journal of Differential Equations, 268(4):1345 - 1378, 2020.

[6] C. Briat. Convex conditions for robust stabilization of uncertain switched systems with guaranteed minimum and mode-dependent dwell-time. Systems and Control Letters, 78:63 - 72, 2015.

[7] Y. Chitour, N. Gugliemi, V.Y. Protasov, and M. Sigalotti. Switching systems with dwell time: Computing the maximal Lyapunov exponent. Nonlinear Analysis: Hybrid Systems, 40:101021, 2021.

[8] Y. Chitour, P. Mason, and M. Sigalotti. On the marginal instability of linear switched systems. Systems 86 Control Letters, 61(6):747-757, 2012.

[9] F.H. Clarke, Y.S. Ledyaev, and R.J. Stern. Asymptotic stability and smooth Lyapunov functions. Journal of Differential Equations, 149(1):69 - 114, 1998.

[10] J. P. Geromel and P. Colaneri. Stability and stabilization of continuous-time switched linear systems. SIAM Journal on Control and Optimization, 45(5):1915-1930, 2006.

[11] R. Goebel and A. R. Teel. Preasymptotic stability and homogeneous approximations of hybrid dynamical systems. SIAM Review, 52(1):87-109, 2010.

[12] J.P. Hespanha and A.S. Morse. Stability of switched systems with average dwell-time. In 38th IEEE Conf. on Decision and Control (CDC), pages 2655-2660, 1999.

[13] H. K. Khalil. Nonlinear Systems. Pearson Education. Prentice Hall, 2002.

[14] A. Kundu, D. Chatterjee, and D. Liberzon. Generalized switching signals for input-to-state stability of switched systems. Automatica, 64:270-277, 2016.

[15] D. Liberzon. Switching in systems and control. Birkhaüser, 2003.

[16] H. Lin and P. J. Antsaklis. Stability and stabilizability of switched linear systems: A survey of recent results. IEEE Transactions on Automatic Control, 54(2):308-322, 2009.

[17] S. Liu, A. Russo, D. Liberzon, and A. Cavallo. Integral-input-to-state stability of switched nonlinear systems under slow switching. IEEE Transactions on Automatic Control, 2020.

[18] S. Liu, A. Tanwani, and D. Liberzon. ISS and integral-ISS of switched systems with nonlinear supply functions. Mathematics of Control, Signals, and Systems, 161, 2021.

[19] A. S. Morse. Supervisory control of families of linear set-point controllers - Part I. exact matching. IEEE Transactions on Automatic Control, 41(10):1413-1431, 1996.

[20] A. Polyakov. Generalized Homogeneity in Systems and Control. Communications and Control Engineering. Springer International Publishing, 2020.

[21] L. Rosier. Homogeneous Lyapunov function for homogeneous continuous vector field. Systems and Control Letters, 19(6):467 - 473, 1992.

[22] R. Shorten, F. Wirth, O. Mason, K. Wulff, and C. King. Stability criteria for switched and hybrid systems. SIAM Review, 49(4):545-592, 2007.

[23] L. Vu, D. Chatterjee, and D. Liberzon. Input-to-state stability of switched systems and switching adaptive control. Automatica, 43(4):639 - 646, 2007.

[24] G.X. Zhang and A. Tanwani. ISS Lyapunov functions for cascade switched systems and sampled-data control. Automatica, 105:216-227, 2019.

[25] X. Zhao, P. Shi, Y. Yin, and S.K. Nguang. New results on stability of slowly switched systems: A multiple discontinuous Lyapunov function approach. IEEE Transactions on Automatic Control, 62(7):3502-3509, 2017. 